\title{
Casting process design and practice for coolant pump impeller in AP1000 nuclear power station
}

\author{
Ping Zhao', "Zhong-li Liu', Gui-quan Wang', Peng Liu' \\ 1. College of Materials Science and Engineering, Qingdao University of Science \& Technology, Qingdao 266044, China \\ 2. School of Nuclear Equipment and Nuclear Engineering, Yantai University, Yantai 264005, China \\ 3. Institute of Metal Research, Chinese Academy of Science, Shenyang 110016, China
}

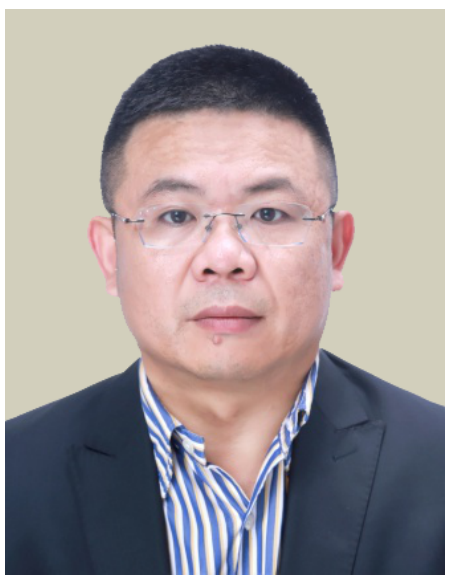

*Zhong-li Liu

Male, born in 1973, Ph.D, Associate Professor. He obtained his Ph.D in Tsinghua University in 2008. His research interests are mainly focused on special steel and its processing technology. He has published about 30 papers in international and domestic journals. $\mathrm{He}$ is now a member of the WFO Ferrous Metals Commission and a member of the Foundry Institution of Chinese Mechanical Engineering Society.

E-mail: liuzhongli@ytu.edu.cn

Received: 2020-01-02

Accepted: 2020-03-03

\begin{abstract}
The coolant pump impeller casting is the only rotating component in the nuclear island of an AP1000 nuclear power station, and is required to have a 60year service time, which requires advanced materials and processing technologies to guarantee. In this paper, the casting process was studied, designed and modified by means of numerical simulation. The gating system was distributed symmetrically and the runner diameter was a little bigger for avoiding sand wash and turbulence; the feeding system focused on the solution of blades feeding, as some parts of which should reach Severity Level 1 radioactive testing standard. Therefore, upper and lower plates cooperating with chillers acted as feeding method besides additional 2-3 times thickness; in addition, lowering sand core strength, decreasing pouring temperature and increasing dimension allowance would be adopted to avoid crack defects. Finally, the pilot impeller was cast. The results show that the casting process design is reasonable, as the liquid rises very smoothly when pouring, and no volume defects are found by means of $100 \%$ radioactive testing. Based on this casting process, 16 coolant pump impellers have been successfully produced and delivered to customers.
\end{abstract}

Key words: AP1000; coolant pump impeller; casting process

CLC numbers: TG142.71; Document code: A; Article ID: 1672-6421(2020)02-173-05

$\mathrm{N}$ uclear power is regarded as a kind of "green energy," and has been put into commercial operation for more than half a century, developed from Generation 1 to Generation $3^{[1-3]}$. Among the Generation 3 nuclear power technologies, the most representative one is the AP1000 developed by Westinghouse of USA. The AP1000 type of reactor is a kind of advanced passive pressurized water reactor (PWR) with a high safety coefficient ${ }^{[4,5]}$. The first four AP1000 nuclear power stations were built in Sanmen County, Zhejiang Province and Haiyang County, Shandong province of China. Among all the components of a nuclear power station, the coolant pump is called the "heart" of the nuclear island, as it supplies power to the coolant system, and the coolant pump impeller is considered the "muscle", which is the only one rotating component driven by a motor and makes the coolant flow ${ }^{[6-8]}$. The coolant pump impeller is required to have a 60 -year service time based on the technical specifications. Because of its complicated shape, casting method is the most commercial method for fabrication of the coolant pump impeller, and sand casting and investment casting processes are often used ${ }^{[9,10]}$. The impellers are mainly made of martensite stainless steels and duplex stainless steels ${ }^{[11-14]}$. The quality requirement of a coolant pump impeller is critical, as it works in the condition of coolant containing neutron, and needs to have the same service time as a nuclear power station, i.e., 60 years. Therefore, only an advanced material and manufacturing process could meet the strict demand. In this study, the casting process was studied in detail, and a pilot impeller was cast. 


\section{Impeller basic information and difficulties analysis}

The impeller and its position in the coolant pump system are shown in Fig. 1. It can be seen that the impeller has a complicated shape with 7 blades that whirl along the upper plate to lower plate. The weight of the impeller is about $352 \mathrm{~kg}$, and the maximum diameter (lower plate) is about $803 \mathrm{~mm}$, with a height of $565 \mathrm{~mm}$.

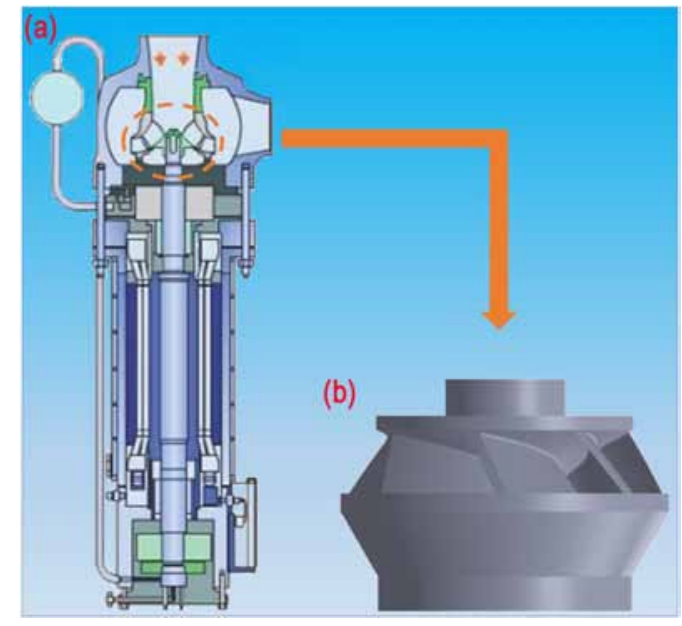

Fig. 1: (a) Profile of the coolant pump system; (b) Impeller

\subsection{Material}

The impeller is made of a low-carbon martensitic stainless steel, which has excellent resistance to corrosion and excellent comprehensive mechanical properties, and is widely used in hydropower station runner and other parts ${ }^{[15-17]}$, e.g., the typical use is the $450 \mathrm{t}$ impeller in the famous Three Gorges hydropower station ${ }^{[18]}$. The nominal chemical composition of the steel is listed in Table 1 (ASTM A-743 CA6NM).

Table 1: Nominal chemical composition of stainless steel (wt.\%)

$\begin{array}{cccccccccc}\text { C } & \mathrm{Cr} & \mathrm{Ni} & \text { Mo } & \text { Co } & \text { Mn } & \text { Si } & \text { P } & \text { S } \\ 0.03-0.06 & 11.5-14.0 & 3.5-4.5 & 0.4-1.0 & \leqslant 0.05 & \leqslant 1.0 & \leqslant 1.0 & \leqslant 0.04 \leqslant 0.03\end{array}$

The liquidus temperature of the low-carbon martensitic stainless steel is about $1,489{ }^{\circ} \mathrm{C}$ and solidus temperature is about $1,470{ }^{\circ} \mathrm{C}$, so the width of the two-phase region is about $20^{\circ} \mathrm{C}$, which means the castability is good. However, as it contains a lot of $\mathrm{Cr}$ which is easy to oxide during melting and pouring, the flowability would be poor without good deoxidation and protection. The material has a good quenchability, and $M_{\mathrm{s}}$ and $M_{\mathrm{f}}$ (start and finish temperature of martensite transformation) are about $230-290{ }^{\circ} \mathrm{C}$ and $70-120{ }^{\circ} \mathrm{C}$, respectively ${ }^{[15]}$, which means even in as-cast state, most of the microstructure would turn to martensite, increasing the crack tendency. Therefore, compared with common carbon steel, the potential oxidation inclusions and crack risk will make it more difficult to make sound castings.

\subsection{Radioactive testing (RT)}

RT is a method to "see" the inner defect, which is not used normally, but only for some very important casting components. In the nuclear island, all the castings should be inspected by RT, but the acceptance criteria are different. Severity Level 1 (China Standard: NB2575.6) represents the highest requirement, and Severity Level 2, a little lower than Level 1. There are 3 regions of the impeller required to reach Severity Level 1, shown in Fig. 2, among which, $\mathrm{A}$ and $\mathrm{C}$ represent a hot spot region, and $\mathrm{B}$ represents a blade region that is $58 \mathrm{~mm}$ length away from the edge. In addition, the left regions are required to reach Severity Level 2. The blade body should be paid much more attention, as part of it is required to reach Severity Level 1, and part of it is Severity Level 2. Once some defects are found in blade, it will be difficult to make a welding repair due to the space limitation. Guaranteeing the soundness of blade is the key point for making a qualified impeller.

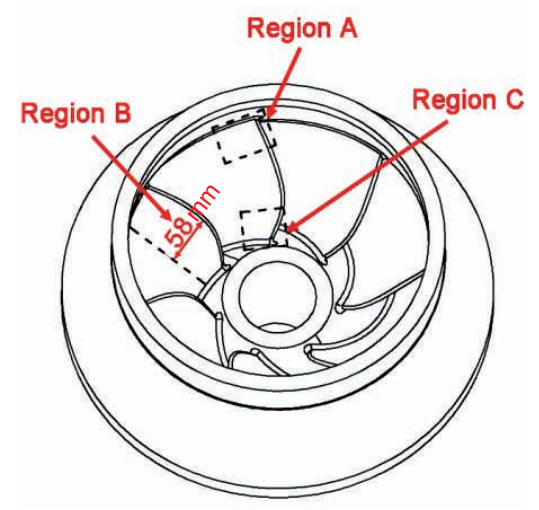

Fig. 2: RT region sketch

\subsection{Blades}

In total, there are 7 blades, and the profile is shown in Fig. 3. The blade has an average arc length of about $423 \mathrm{~mm}$, height of about $265 \mathrm{~mm}$, and thickness of about $8 \mathrm{~mm}$.

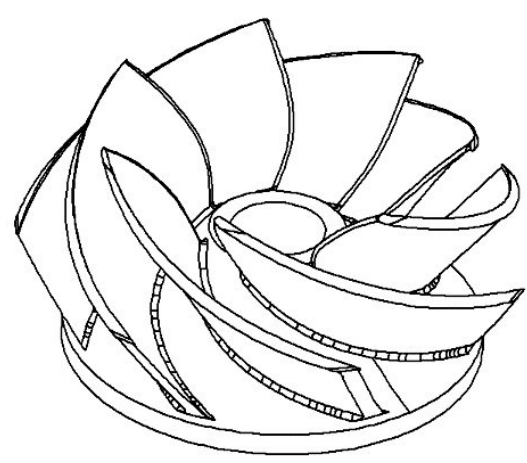

Fig. 3: Blade profile

For sand casting, each material has a minimum permitted wall thickness because of the liquid surface tension, and for stainless steel, the value is $8-10 \mathrm{~mm}{ }^{[19]}$. Therefore, to make a sound blade without welding repair seems difficult, as the blade thickness is near the material limitation, in addition, 
it is difficult to feed the blade by risers. The two ends of the blade are connected with the upper and lower plate, and the junction (Regions A and C in Fig. 2), i.e., the hot spot, should be fed by side risers and top risers, respectively. However, it is impossible for the top and side risers to satisfy the whole blade feeding because of the feeding distance limitation of each riser, and it is also difficult to find other special postitions to place risers to feed. Therefore, strict RT criteria and inherent design difficulty are big obstacles for impeller.

\subsection{Crack}

When liquid steel cools down and solidifies, stress would appear within the castings, and the more complicated the shape, the greater the stress. In addition, if brittle microstructure exists, it would increase crack possibility. So, the coolant pump impeller is easy to crack.

\section{Casting process design}

Generally, casting process design includes the design of the gating system and the feeding system. According to the analysis above, some special points should be paid attention when creating the casting process design.

\subsection{Gating system}

The gating system should be not only in favor of filling, but also prevent liquid from oxidation. Therefore, the inner runner should be distributed symmetrically to make the liquid flow smoothly, in addition, the runner diameter should be a little bigger to decrease the liquid speed, so as to avoid sand wash and turbulence. The sketch of a gating system is shown in Fig. 4.

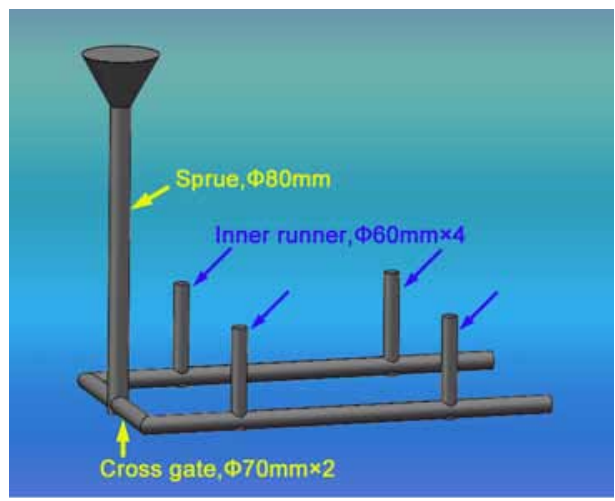

Fig. 4: Gating system

\subsection{Feeding system}

As shown in Fig. 2, there are two hot spot regions, Regions $\mathrm{A}$ and $\mathrm{C}$, which would be fed by side riser and top riser, respectively, as shown in Fig. 5. However, it is difficult to find an area to place risers to feed the blades. In order to guarantee the blade soundness, these measures should be taken: firstly, the blade thickness should be increased to 2-3 times; secondly, some chillers would be placed in the middle of blade width (shown in Fig. 6). The area above the chillers would be fed by an upper plate, while the area below the chillers would be fed by a lower plate. The feature of such design is to feed the casting by itself.

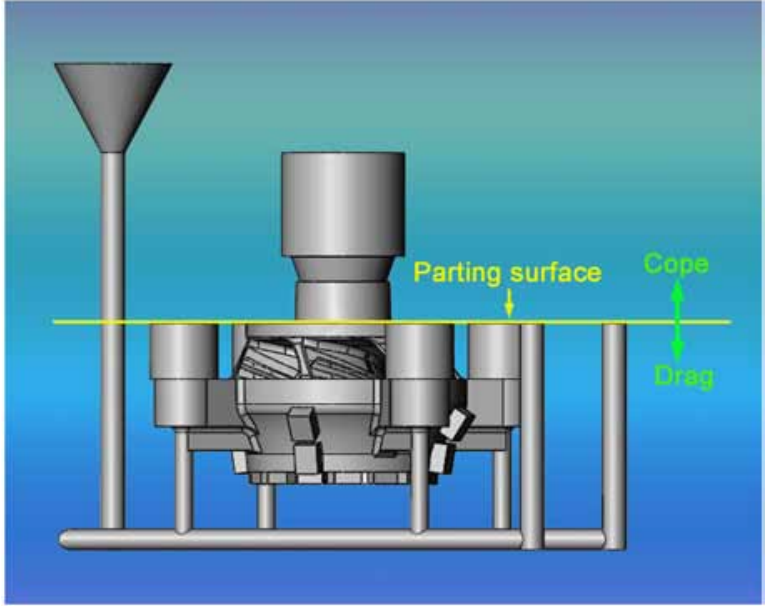

Fig. 5: Feeding system

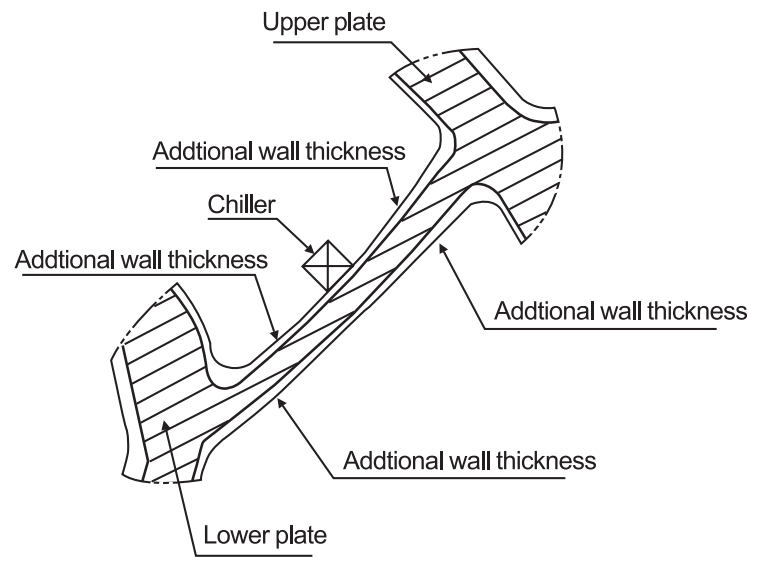

Fig. 6: Sketch of blade feeding

\subsection{Crack prevention}

In order to avoid crack, firstly, the sand core strength should be less than the mold by means of modification of resin binder content; secondly, the pouring temperature should be controlled in a narrow range; thirdly, proper allowance should be added on the casting.

\section{Numerical simulation}

In order to verify the reasonability of the casting process, simulation was made by means of ProCast software. The liquidus temperature of the steel is about $1,489{ }^{\circ} \mathrm{C}$, and thus the pouring temperature is set to $1,570{ }^{\circ} \mathrm{C} \pm 10^{\circ} \mathrm{C}$ according to experience.

The filling simulation results of the impeller are shown in Fig. 7. At the beginning of filling, the liquid was injected from the gate with a pouring speed of about $90 \mathrm{~kg} \cdot \mathrm{s}^{-1}$. When $t=3.24$ $\mathrm{s}$ (Fig. 7a), the liquid filled the cross gate and inner runner, and began to fill the mould cavity. When $t=6.44 \mathrm{~s}$ (Fig. 7b), the liquid filled the mould cavity, and the liquid filling speed was very fast because the blade wall was thin, thus the impact of the high temperature liquid on the cavity wall was severe. When $t=9.73 \mathrm{~s}$ (Fig. 7c), the blade and lower cover parts were almost completely full and the upper cover started to be filled. When $t=17.45 \mathrm{~s}$ (Fig. 7d), the filling process was completed. Figure 7 only shows some pictures at different times, and does not clearly 


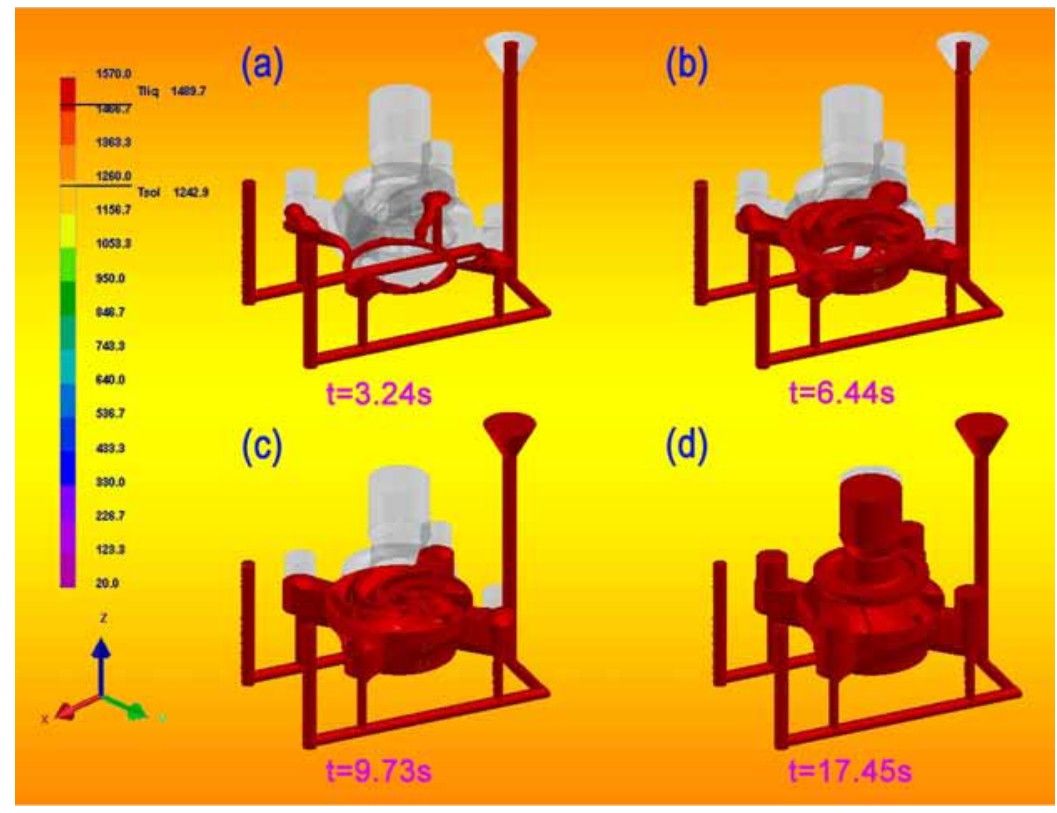

Fig. 7: Simulation of filling process

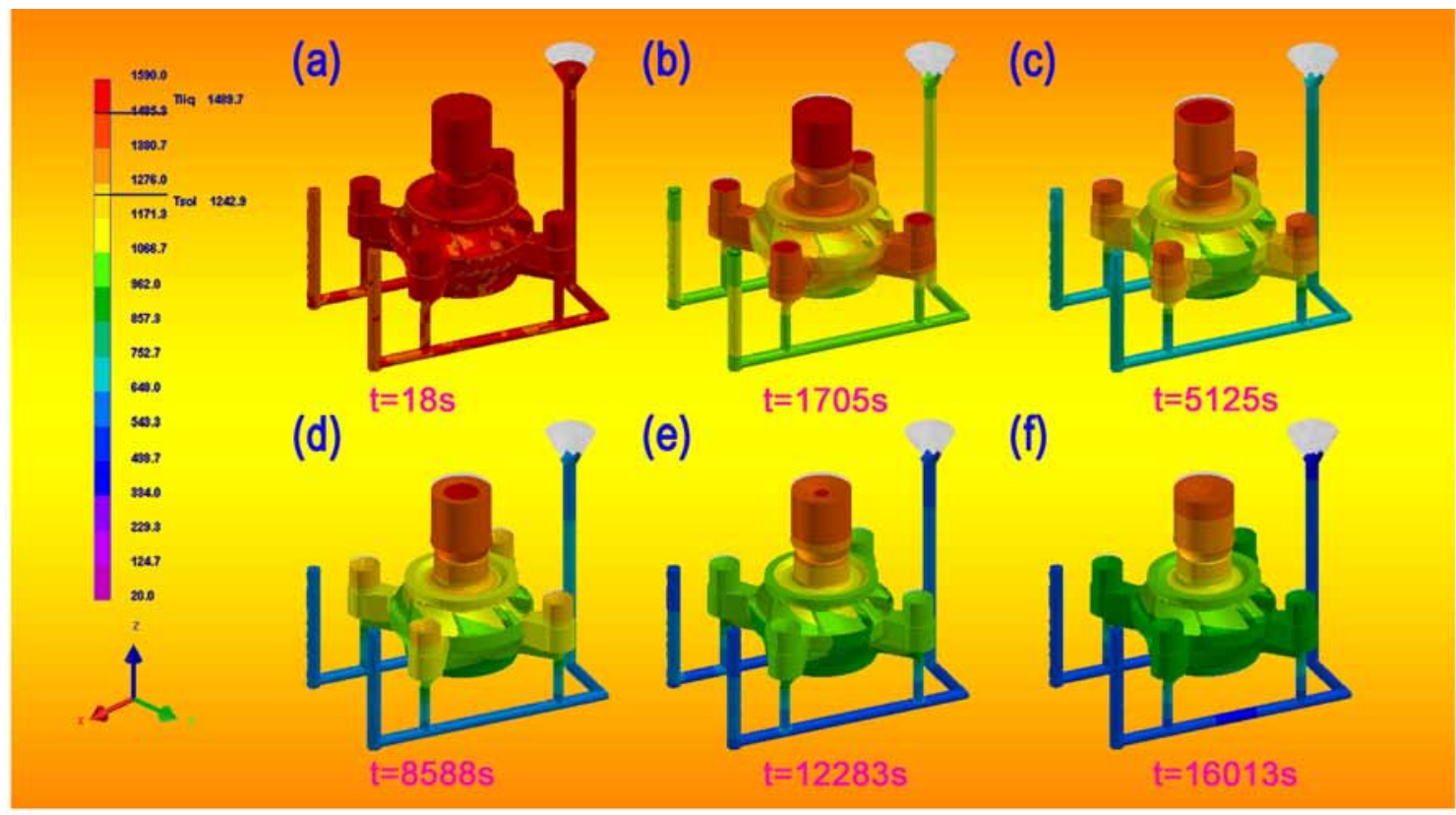

Fig. 8: Simulation of solidification process

describe a filling process. In fact, the filling process video shows that the liquid level rises very smoothly, and the liquid filling order is reasonable.

The temperature field during solidification is shown in Fig. 8. When $\mathrm{t}=18 \mathrm{~s}$ (Fig. 8a), the filling process was completed, and then solidification started. It could be seen that the impeller solidified from bottom to top, and the final solidification regions are risers, so all the defects should exist in the risers.

In terms of the inherent porosity determination method of ProCast software, it could forecast the distribution of shrinkage and cavity defects. The result is shown in Fig. 9. It can be seen that all the shrinkage and cavity defects exist in the risers, and there are no such defects in the impeller itself, indicating that the casting process design is by and large reasonable.

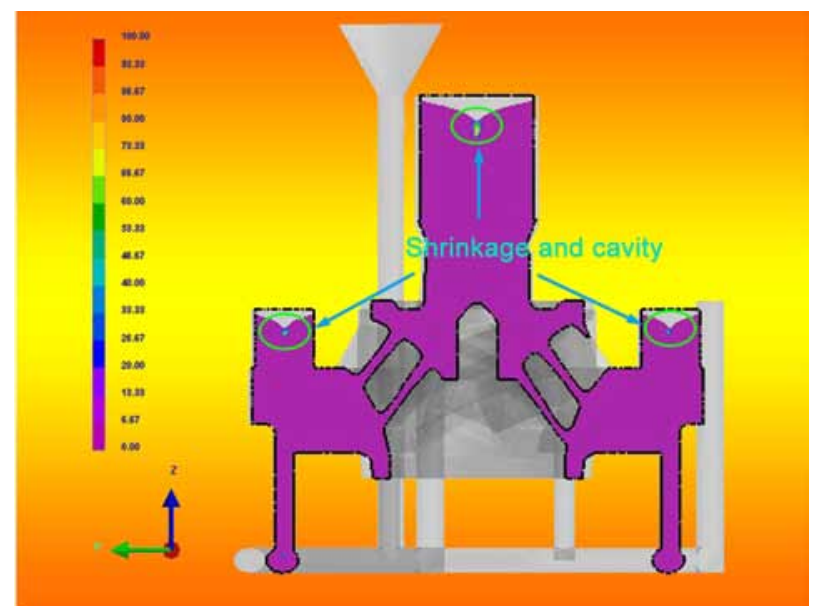

Fig. 9: Possibilities of shrinkage and cavity sites 

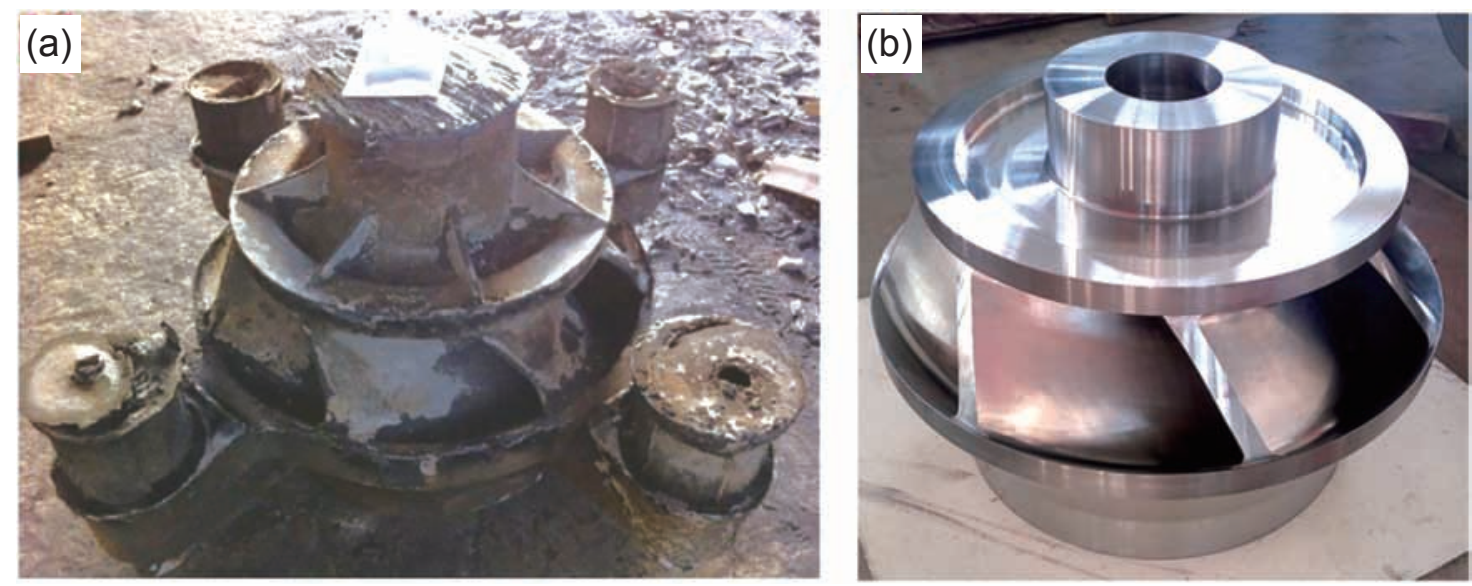

Fig. 10: Pilot impeller: (a) as-cast; (b) after machining

\section{Production}

To validate the simulation results, a pilot production was made. The final impeller is shown in Fig.10. There are no cracks in the impeller, indicating that the measures adopted to decrease stress are effective. The radioactive testing shows no unqualified region, proving the casting process is reasonable. At this point, 16 impellers have been delivered to customers.

\section{Conclusions}

The following conclusions can be drawn:

(1) For the coolant pump impeller, lowering sand core strength, decreasing pouring temperature, and increasing dimension allowance are effective ways to avoid crack.

(2) For the complicated impeller, the gating system should be distributed symmetrically and the runner size should be a little bigger to avoid sand wash and turbulence.

(3) In order to guarantee the blade soundness, 2-3 times thickness allowance and effective feeding are necessary.

\section{References}

[1] Ioannis $\mathrm{N} \mathrm{K}$. The future of the nuclear industry reconsidered: risks, uncertainties and continued promise. Energy Policy, 2012, 48: 185-208.

[2] Mariangela G, Renato G. A nuclear power renaissance? Technological Forecasting \& Social Change, 2012, 79: 1746-1760.

[3] Steve T. Competitive energy markets and nuclear power: Can we have both, do we want either? Energy Policy, 2010, 38: 4903-4908.

[4] Regis A M. AP1000 will meet the challenges of near-term deployment. Nuclear Engineering and Design, 2008, 238: 1856-1862.

[5] Schulz T L. Westinghouse AP1000 advanced passive plant. Nuclear Engineering and Design, 2006, 236: 1547-1557.

[6] Wang Xiuli, Lu Yonggang, Zhu Rongsheng, et al. Study on bidirectional fluid-solid coupling characteristics of reactor coolant pump under steady-state condition. Nuclear Engineering and Technology, 2019, 51(7): 1842-1852.
[7] Zuo Xuebing, Chen Jingjing, Zhang Jindong, et al. Installation of main equipments of reactor coolant system in AP1000 nuclear power Plant. Pressure Vessel Technology, 2013, 11: 62-69. (In Chinese)

[8] Zhu Rongsheng, Liu Yong, Wang Xiuli, et al. The research on AP1000 nuclear main pumps' complete characteristics and the normalization method. Annals of Nuclear Energy, 2017, 99: 1-8.

[9] Zhao Jian, Yi Chunlei. Optimization of investment casting process for stainless steel impeller with complicated geometry. Chinese Journal of Materials Research, 2015,2 9(12): 955960. (In Chinese)

[10] Zhan Chuangang. Production of super duplex stainless steel impeller. Foundry Technology, 2018, 39(9): 1972-1974. (In Chinese)

[11] Wang Guiquan, Liu Zhongli, Sun Zhiqiang, et al. Numerical simulation and optimization of casting method of duplex stainless steel impeller for nuclear power. Foundry, 2012, 61(10): 1146-1151. (In Chinese)

[12] Zhang Jiyan, Liu Zhongli, Wang Guiquan. Casting process for impeller of seawater deep well pump in nuclear power plant. Foundry Technology, 2015, 36(2): 545-548. (In Chinese)

[13] Huang Hongjun, Cao Yang, Wang Haoqiang, et al. Casting process design and optimization of martensite stainless steel pumping impeller. Foundry, 2015, 64(4): 317-320. (In Chinese)

[14] Deng Xuelin. Development of super duplex stainless steel impeller. Foundry Technology, 2014, 35(4): 752-754. (In Chinese)

[15] Song Yuanyuan, Li Xiuyan, Rong Lijian, et al. The influence of tempering temperature on the reversed austenite formation and tensile properties in $\mathrm{Fe}-13 \% \mathrm{Cr}-4 \% \mathrm{Ni}-\mathrm{Mo}$ low carbon martensite stainless steels. Materials Science and Engineering A, 2011, 528: 4075-4079.

[16] Huang Hongjun, Cao Yang, Wang Haoqiang, et al. Casting process design and optimization of martensite stainless steel pumping impeller. Foundry, 2015, 64(4):317-320. (In Chinese)

[17] Song Yuanyuan, Li Xiuyan, Rong Lijian, et al. Anomalous phase transformation from martensite to austenite in $\mathrm{Fe}$ $13 \% \mathrm{Cr}-4 \% \mathrm{Ni}-\mathrm{Mo}$ martensitic stainless steel. Journal of Materials Science \& Technology, 2010, 26(9): 823-826.

[18] Liu Baoxi, Wang Houshi, Yang Jiwei. Study on crown, band and blade of hydraulic turbine runner for three gorges project. Foundry, 2013, 62(7): 673-678. (In Chinese)

[19] Li Rongde, Mi Guofa. Casting process. Beijing: China Machine Press, 2013: 97-111. (In Chinese) 